\title{
Transient correction of the LW-ISOCAM data for low contrasted illumination
}

\author{
A. Coulais and A. Abergel \\ Institut d'Astrophysique Spatiale, Bâtiment 121, Université Paris XI, 91405 Orsay Cedex, France
}

Received May 25; accepted September 15, 1999

\begin{abstract}
The detector of the Long Wavelength channel of the ISOCAM camera on-board the Infrared Space Observatory (ISO) presents systematic memory effects of the response which can bias the photometry by a factor as high as $40 \%$. We show that, for uniform illumination, the non linear model developed by Fouks and Schubert (1995) for the detector of PHT-S channel of the ISOPHOT instrument allows us to describe the LW-ISOCAM response to upward and downward steps of flux with an accuracy better than $1 \%$. For each pixel of the detector, only two parameters have to be used. These parameters are constant over the whole life of ISO. On the other hand, spatial variations from pixel to pixel are significant.

We present an inversion method of the Fouks-Schubert model. Without any fitting and for low contrasted illumination, data are corrected with a photometric accuracy of $\sim 1 \%$, which is typically ten times better than previous methods. We suggest that the same approach could be used for others ISO detectors, and in the future for other experiments.
\end{abstract}

Key words: instrumentation: detectors - methods: data analysis — methods: numerical

\section{Introduction}

Because of the low brightness of the sky and their very low operating temperatures (typically a few K), the extrinsic photo-conductors used in infrared astronomy generally present systematic memory effects of the response. The response after a flux variation is not instantaneous and strongly depends on what has been observed before. The response can never be stabilized, and the final photometry is biased by a factor as high as several $10 \%$. Systematic corrections of these transient effects are generally necessary (1) to remove the trails due to the displacement of

Send offprint requests to: A. Coulais bright sources (2) to improve the photometric accuracy and (3) to reduce the observing times.

All the detectors of the four instruments on board the ISO satellite (Kessler et al. 1996) are extrinsic photoconductors and are affected by this problem at various levels. Several examples of those transient effects have been published for the different detectors of ISO (see Table 1). The response curves are classified in three main families from the behavior after upward step of flux. A detector has a "normal" behavior if the response to an instantaneous increase of the input flux increases monotonously after a quasi instantaneous jump. If the response presents a quasi instantaneous jump followed by a decrease and then by a monotonous increase, we have a "hook" behavior (Fouks 1992). The detector of the SW channel of ISOCAM has an "other" behavior since it does not present any instantaneous jump (but only a monotonous increase).

The ISOCAM camera (Cesarsky et al. 1996) operates in the $2.5-18 \mu \mathrm{m}$ range using two channels, the Short Wavelength one (SW) going from 2.5 to $5.5 \mu \mathrm{m}$, and the Long Wavelength one (LW) from 4 to $18 \mu \mathrm{m}$. The channels are selected using a wheel holding Fabry mirrors. The observing configuration is defined for each channel with a lens wheel to select the field of view per pixel $(1.5,3,6$ and $\left.12^{\prime \prime}\right)$, and a filter wheel to select the spectral band pass. The operating temperature of the camera in flight is around $3 \mathrm{~K}$. The integration times allowed in flight are $0.28,2 ., 5 ., 10$. and 20. s.

This paper is focused on the LW channel of ISOCAM. The filter wheel of this channel contains 10 broad band filters and two Continuously Variable Filters (CVF). The detector is a single crystal made of Si:Ga photo-conductor connected by Indium bumps to direct voltage readout circuits (Agnèse et al. 1989; Cesarsky 1992). $32 \times 32$ square pixels are electrically defined with a pitch of $100 \mu \mathrm{m}$. The crystal is $500 \mu \mathrm{m}$ thick. The doper concentration of the bulk $p$ is $\sim 5.10^{16} \mathrm{~cm}^{-3}$. The temperature inside the bulk is $\sim 3 \mathrm{~K}$. The applied voltage was $\sim 20 \mathrm{~V}$ during the flight. The precise characteristics of the $p^{+}$contacts are not known. The quantum efficiency is $\sim 0.25$. 
Table 1. Table of main families of low background IR detector (non exhaustive) on board ISO satellite. See type definition in Sect. 1

\begin{tabular}{lll}
\hline Type & Detector & Reference \\
\hline normal & CAM Si:Ga LW & (Pérault et al. 1994; Abergel et al. 1999) \\
& PHT Si:Ga linear detector of PHT-S & (Fouks \& Schubert 1995) \\
& SWS Ge:Be and Si:Ga single detectors of the grating section & (Wensink et al. 1992) \\
& LWS Ge:Be detectors of channel SW1 and stressed Ge:Ga of LW2 & (Church et al. 1992b; Church et al. 1992a) \\
\multirow{2}{*}{ hook } & PHT Si:Ga single detector of the channel P1 & (Groezinger et al. 1992) \\
& SWS Si:Sb single detectors of the Fabry-Pérot section & (Wensink et al. 1992b; Church et al. 1992a) \\
& LWS unstressed Ge:Ga detector of the LW1 channel & (Church et al. 1992b; Church et al. 1992a) \\
other & CAM In:Sb SW & (Tiphène et al. 1999) \\
\hline
\end{tabular}

The transient behavior of the LW channel has two components (Abergel et al. 1999): a short-term one which affects typically $40 \%$ of the total step and a very long-term drift which affects typically $5-10 \%$ of the flux. Actually, the long-term component is not fully understood (we do not know whether it is predictable) and there is no method to correct it without using the redundancy of the observations (Miville-Deschênes et al. 1999). By contrast the short-term transient is perfectly predictable for uniform illuminations, and we present in Sect. 2 its main properties. In several cases (especially near the dark level), upward and downward steps are extremely different, which strongly constrains the models of the response used to develop correction algorithms. The model which has been used during the first two years of the mission, from 1996 to 1998 , to process most of the data (Abergel et al. 1996) is not able to produce the observed asymmetry.

From a theoretical point of view, the Suris-FouksVinokurov team has studied low background photo detectors during the last two decades (Suris \& Fouks 1978; Suris \& Fouks 1980; Fouks 1981a; Fouks 1981b; Vinokurov \& Fouks 1991; Fouks 1992; Fouks 1996). Using solid state and semiconductor physics, they have derived several analytical models from the physical equations. The key parameters for such a detector are the doper concentration of the bulk $p$, the thickness of the bulk, the temperature inside the bulk, the contact properties (the dopant concentration of the $p^{+}$contacts) and the applied voltage. One very important hypothesis in these models is that the illumination of the pixel surface is uniform.

We describe in Sect. 3 one of these models developed by Fouks and Schubert (1995) for the Si:Ga detector of the PHT-S channel of ISOPHOT. Because several approximations can be made for this kind of detector, this model is one of the simplest non-linear models that Vinokurov \& Fouks (1991) have developed. In Sect. 3, we show that this model allows us a remarkable adjustment of experimental data obtained for uniform illumination of the LW channel of ISOCAM, both for upward and downward steps. Each pixel is characterized by only two parameters (Sect. 3.5). We propose in Sect. 4 an original inversion method to correct systematically the data without any fitting. Limitations and works in progress are described in Sect. 5. Finally, we suggest in Sect. 6 extending our approach to other extrinsic photo-conductors of ISO and further experiments (especially to the normal ones, see Table 1).

\section{ISOCAM transients description}

\subsection{Global description}

The transient behavior of the LW ISOCAM detector has been analyzed during ground-based observations at IAS (Pérault et al. 1994; Abergel \& Pérault 1994) and the current understanding after the mission is detailed in Abergel et al. (1999). We see in Fig. 1 that going from the dark level to an uniform illumination, the response is made up of a short-term transient followed by a long-term drift, with typical time constants equal to one minute and one hour respectively. This paper is focused on the short term transient.

The short term transient is made (1) of a jump of about $60 \%$ of the total step and (2) of a signal behavior which depends on the flux history, the amplitude of the current step, the pixel position on the detector matrix and the local spatial gradient illumination (Abergel et al. 1999). Upward and downward steps are not symmetrical (compare Figs. 2 and 3). The model systematically used up to now to process most of the data (Abergel et al. 1996) does not produce any asymmetry in the response curve, so the final precision cannot be better than $\sim 10 \%$.

The short term transient is extremely reproducible (the same history gives exactly the same response curve), so experimental data give very strong constrains for new models.

\subsection{Short transient asymmetry}

The asymmetry of the response is analyzed using data obtained during the preflight characterization (Pérault et al. 1994) and the revolution 16 of the Performance Verification phase (PV phase) of ISO. Uniform extended fields were observed (integrated spheres during preflight test, zodiacal background during revolution 16). The data 

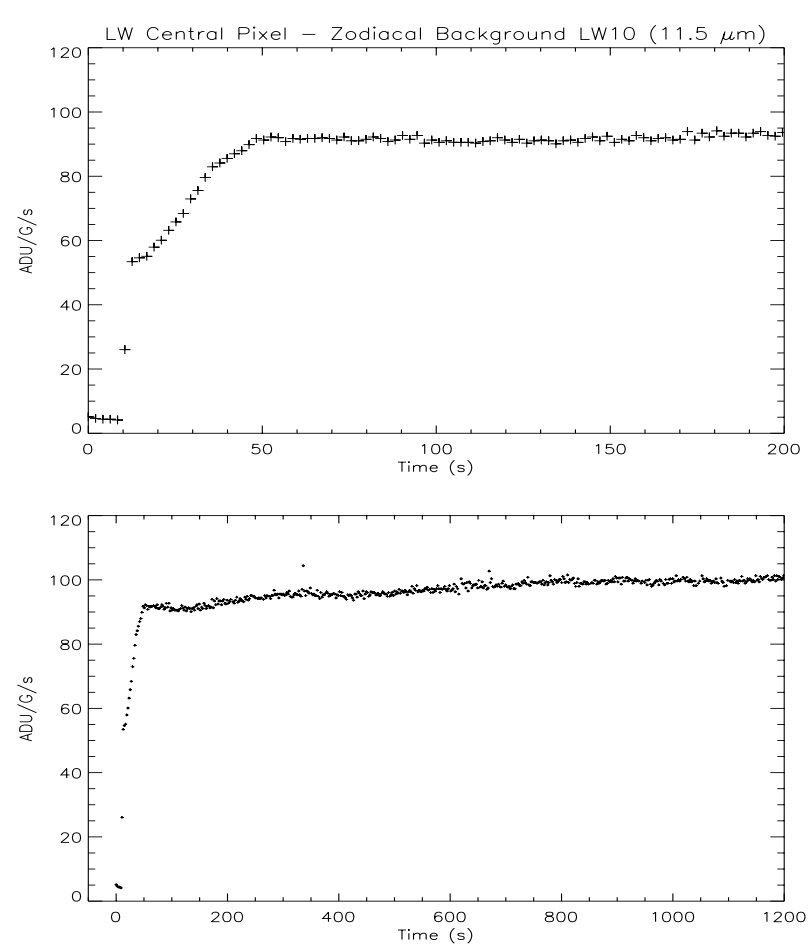

Fig. 1. Response of the central pixel after a flux step going from the dark level to the zodiacal emission observed with the LW10 broad-band filter $(8-15 \mu \mathrm{m}$ and centered to $11.5 \mu \mathrm{m}$, coinciding to the $12 \mu \mathrm{m}$ IRAS band). The integration time of each frame is $2.1 \mathrm{~s}$. This observation was taken at the beginning of a revolution of ISO, just after the switching on of the camera. Upper panel: First frames showing the short-term transient (see the text). Lower panel: Whole observation showing the long-term drift. (Fig. from Abergel et al. 1999)

presented in this paper correspond to individual pixels or mean values inside the $11 \times 11$ central square. Moreover, the signals measured by the camera are given using the internal output units of the camera ("ADU": Analog to Digital Unit) divided by the electronic gain and the integration time (units: ADU/G/s). To convert these numbers into physical units, see Blommaert (1998). For example, with the LW 1 filter, $1 \mathrm{ADU} / \mathrm{G} / \mathrm{s} \simeq 1 \mathrm{mJy}$.

The response to upward step from an initial level close to the dark level to a high level of illumination presents an inflection point (Fig. 2). This inflection point appears for all observations starting from levels not too far from the dark level and going to a final flux above $\sim 5 \mathrm{ADU} / \mathrm{G} / \mathrm{s}$. Its temporal position strongly depends on the initial and final fluxes (see also Sect. 3.2). For small steps of flux (typically below $\sim 10 \mathrm{ADU} / \mathrm{G} / \mathrm{s}$ ) this inflection point can be hidden by the noise. For upward steps higher than $\sim 50 \mathrm{ADU} / \mathrm{G} / \mathrm{s}$, a long term drift may also be visible (see Fig. 1). For a low ratio of steps of fluxes, this inflection point does not exist.

For downward steps from a high and uniform illumination to the dark level (Fig. 3), the inverse of the re-
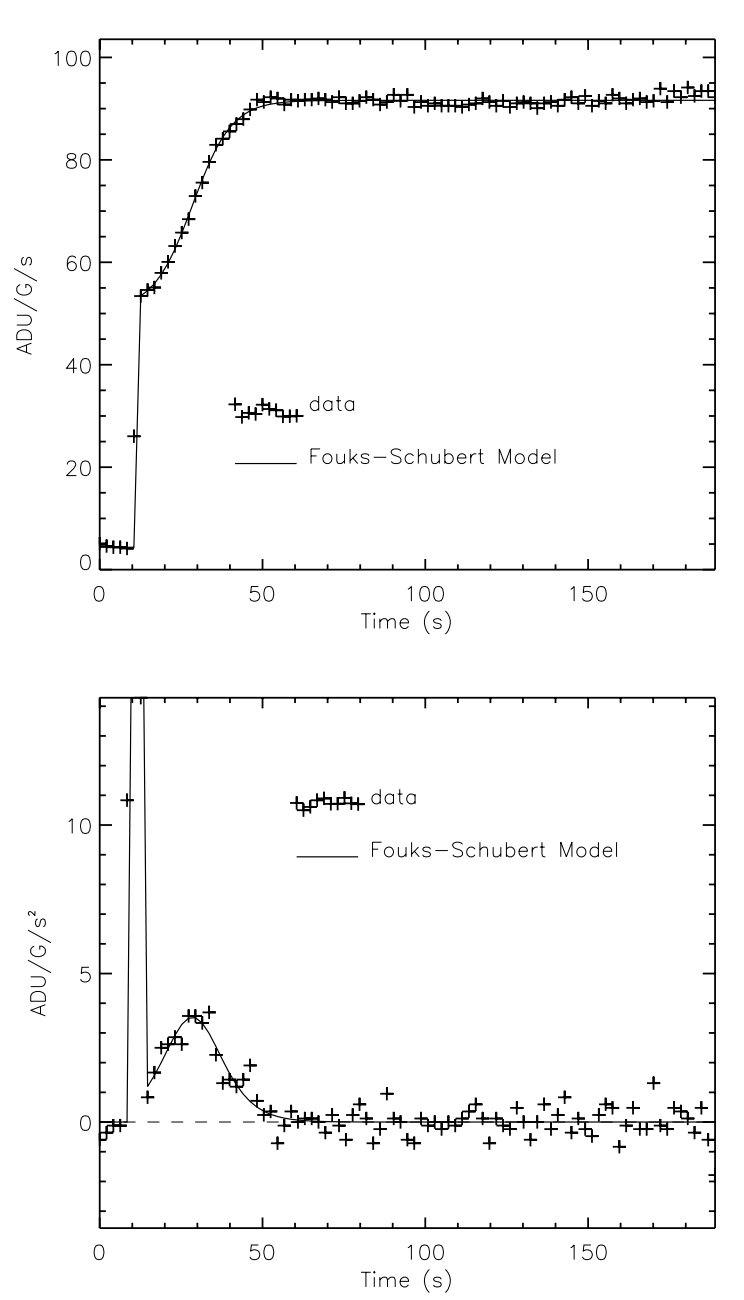

Fig. 2. Upward step of flux. These data were take during inflight observations (tdt 12900101). Upper panel: Data (+, central pixel) and the Fouks-Schubert model (solid line) in linear scales. Lower panel: Derivative of the data $(+)$ and the model (solid line). The levels before the step of flux is constant and stabilized. Therefore, we used Eq. (1), with: $\beta=0.55, \lambda=551$. $\mathrm{ADU} / \mathrm{G}, J_{0}^{\infty}=4.4 \mathrm{ADU} / \mathrm{G} / \mathrm{s}$ and $J_{1}^{\infty}=91.6 \mathrm{ADU} / \mathrm{G} / \mathrm{s}$. In the difference between the data and the model, we can see the errors are below $\sim 3 \%$

sponse increases linearly with time. So, the general shape of the downward steps is hyperbolic rather than exponential. The value of the slope depends on the initial flux. There is neither an inflection point nor long term drift.

Finally, ground-based data have indicated that the time constants (up and down) for each pixel and for any step of flux are at a first order inversely proportional to the current illumination.

\subsection{Modeling of the short-term transient}

A lot of models have been already proposed to describe ISOCAM-LW transients. The simplest ones use simple 

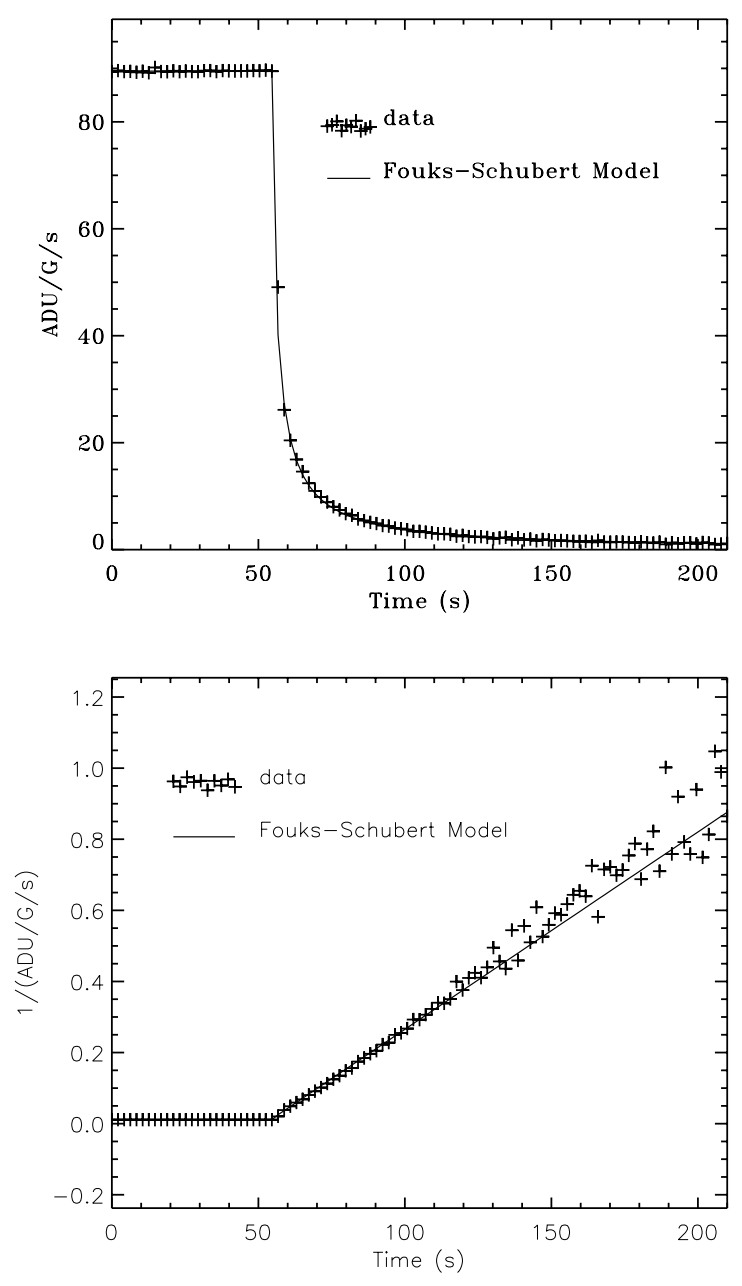

Fig. 3. Downward step to dark level (data taken during the preflight measurements). Upper panel: mean central pixel $(+)$ and Fouks-Schubert model (line) in linear scales. Lower panel: inverse of the data $(+)$ and the model (line) in order to show the quasi linear shape of the downward step. The level before the flux change is constant and stabilized. The level after is the dark level (very close to zero). Therefore we used Eq. (2), with: $\beta=0.55, \lambda=400$. ADU $/ \mathrm{G}, J_{0}^{\infty}=90 . \mathrm{ADU} / \mathrm{G} / \mathrm{s}$ and $J_{1}^{\infty}=0$. ADU $/ \mathrm{G} / \mathrm{s}$. Preflight and in-flight values of $\lambda$ and $\beta$ are different (compare with 2) due to a different setup of the detector

exponential formulae to describe the response starting from a stabilized level to a new one (e.g. Delattre et al. 1996). However, such an approach generally fails because (1) it does not take into account the whole history of illumination and (2) observed upward and downward responses are not symmetrical.

In previous studies (Abergel et al. 1996; Abergel et al. 1999), we have proposed an integral description of the response to take into account the history of illumination. This model ("the old IAS model") has been systematically used during the last two years, but it fails to reproduce the asymmetry. Finally non linear models (see for instance (Ganga et al. 1998b; Ganga et al. 1998a) and the IRA model (Lari 1997) are more promising. However at the present time, none of these models have been systematically tested and compared with ISOCAM observations.

\section{The Fouks-Schubert model (FS model)}

\subsection{Origin of the model}

The Si:Ga linear detector of the PHT-S channel of ISOPHOT has been extensively studied by Fouks and Schubert from experimental and theoretical points of view (Fouks 1992; Schubert et al. 1994; Fouks \& Schubert 1995; Schubert et al. 1995; Schubert 1995). The LW ISOCAM detector is a Si:Ga array too. Its size is $32 \times$ 32 pixels, $100 \mu \mathrm{m}$ pitch and the thickness of $500 \mu \mathrm{m}$. PHT-S pixels size are $0.35 \times 0.37 \mathrm{~mm}$ (for 64 pixels long) and the thickness is $2.0 \mathrm{~mm}$. Wavelength ranges are 6.12. $\mu \mathrm{m}$ for PHT-S and 4.-17. $\mu \mathrm{m}$ for LW ISOCAM.

In this paper, we will not describe the semi conductor physics equations but only indicate the more relevant references. Because of the intrinsic non linear nature of the equations and of the sensitivity to the boundary conditions, several steps were needed from the first linearized solutions (Suris \& Fouks 1978; Suris \& Fouks 1980) to the final non linear solutions covering a large range of detectors (Vinokurov \& Fouks 1991; Fouks 1992). For the detectors of PHT-S and LW-ISOCAM, the most relevant paper to understand the physics is Fouks \& Schubert (1995). It is based on the first order description of the physics inside the bulk of an extrinsic $p$-type photo-conductor. After several hypothesis about the dominant physical processes, a set of conditions and differential equations is obtained. Solving the set of differential equations assuming a constant electric field inside the bulk, a Riccati differential equation is found. Its solution which gives the temporal response is a non linear equation.

\subsection{The equations}

The first solution describes the response to a instantaneous step of flux at time $t=0$, from the constant level $J_{0}^{\infty}$ to the constant level $J_{1}^{\infty}$ :

$J(t)=\beta J_{1}^{\infty}+\frac{(1-\beta) J_{1}^{\infty} J_{0}^{\infty}}{J_{0}^{\infty}+\left(J_{1}^{\infty}-J_{0}^{\infty}\right) \exp (-t / \tau)}$

$J_{1}^{\infty}$ is the stabilized photo-current measured at time $t=$ $+\infty$. It is also directly related to the observed flux, since a linear relationship is assumed between the flux and the photo-current after stabilization (Fouks \& Schubert 1995). The parameter $\beta$ characterizes the instantaneous jump just after the flux change. The theory gives a simple relationship between the time constant $\tau$ and $J_{1}^{\infty}$ over several order of magnitude: $\tau=\lambda / J_{1}^{\infty}$. Yet, the time constant is $\lambda$. This dependence has also been observed for the LW 
channel of ISOCAM (Sect. 3.4 and Abergel et al. 1999). Equation (1) does not allow us to compute $J(t)$ if $J_{1}^{\infty}=0$, and for that case a first order development gives:

$J(t)=\frac{(1-\beta) J_{0}^{\infty}}{1+\frac{t}{\lambda} J_{0}^{\infty}}$.

We can see in Fig. 3 that the agreement of Eq. (2) with the data is perfect.

On data (ground based and in-flight data), starting from a level close to the dark level (lower than $1 \mathrm{ADU} / \mathrm{G} / \mathrm{s}$ ), an inflection point (see Sect. 2 and Fig. 2) is clearly visible for moderate upward steps (typically above $10 \mathrm{ADU} / \mathrm{G} / \mathrm{s}$ and below $100 \mathrm{ADU} / \mathrm{G} / \mathrm{s}$ ). It is more difficult to observe it for strong upward steps since it is temporally very close to the jump. No inflection point is visible for small upward steps (ratio $J_{1}^{\infty} / J_{0}^{\infty}$ between 1 and 2) and for downward steps. This inflection point is predicted and perfectly reproduced with Eq. (1). Its position $t^{\text {ip }}$ can be easily calculated from Eq. (1). For $J_{0}^{\infty}>0$, we obtain:

$t^{\mathrm{ip}}=\frac{\lambda}{J_{1}^{\infty}} \times \ln \left(\frac{J_{1}^{\infty}-J_{0}^{\infty}}{J_{0}^{\infty}}\right)$.

Much information comes from this equation. An inflection point is not predicted for downward steps $\left(\left(J_{1}^{\infty}-J_{0}^{\infty}\right)<\right.$ $0)$. The inflection point appears for upwards steps only when $J_{1}^{\infty} / J_{0}^{\infty}>2$. For $J_{0}^{\infty}=0, t^{\text {ip }}=+\infty$, so in that case the response is constant and, $\forall t>0, J(t)=\beta J_{1}^{\infty}$. However the case $J_{0}^{\infty}$ strictly equal to 0 (and obviously $J_{0}^{\infty}<0$ ) is not physical, since the detector always receives infrared photons due to thermal emissions of the different parts of the camera (even when it is switched off). For $J_{0}^{\infty}>0$, Fig. 4 illustrates the variations of the position of the inflection point with the value of the initial level $J_{0}$.

\subsection{From two non stabilized levels}

Equation (1) can only be used for transitions between stabilized levels. The following enhancement (Fouks \& Schubert 1995) allows us to describe a not stabilized detector output $J_{n}(t)$ from a recursive point of view (see Fig. 5 for notations). An observation is made of $N$ integrations starting at time $t_{n}$. We assume that the input flux $J_{n}^{\infty}$ is constant during each successive integration. During the integration number $n$, we have:

$J_{n}(t)=\beta J_{n}^{\infty}+\frac{(1-\beta)\left(J_{n}^{\text {ini }}-\beta J_{n}^{\infty}\right) J_{n}^{\infty}}{J_{n}^{\text {ini }}-\beta J_{n}^{\infty}+\left(J_{n}^{\infty}-J_{n}^{\text {ini }}\right) \mathrm{e}^{\left(-\left(t-t_{n}\right) / \tau_{n}\right)}}$.

Basically we use the same notations as in Eq. (1). $J_{n}^{\text {ini }}$ is the response measured just after the jump at time $t_{n}$. $J_{n}(t)$ is the response at any time $t$ between $t_{n}$ and $t_{n+1}$. In this equation, the state at the end of the previous integration $n-1$ is contained in $J_{n}^{\text {ini }}$, which can be related to the previous flux and the current stabilized flux values $\left(J_{n-1}^{\infty}\right.$ and $\left.J_{n}^{\infty}\right)$ and the final previous flux just before the jump $J_{n-1}^{\text {end }}$ by:

$J_{n}^{\text {ini }}=J_{n-1}^{\text {end }}+\beta\left(J_{n}^{\infty}-J_{n-1}^{\infty}\right)$.

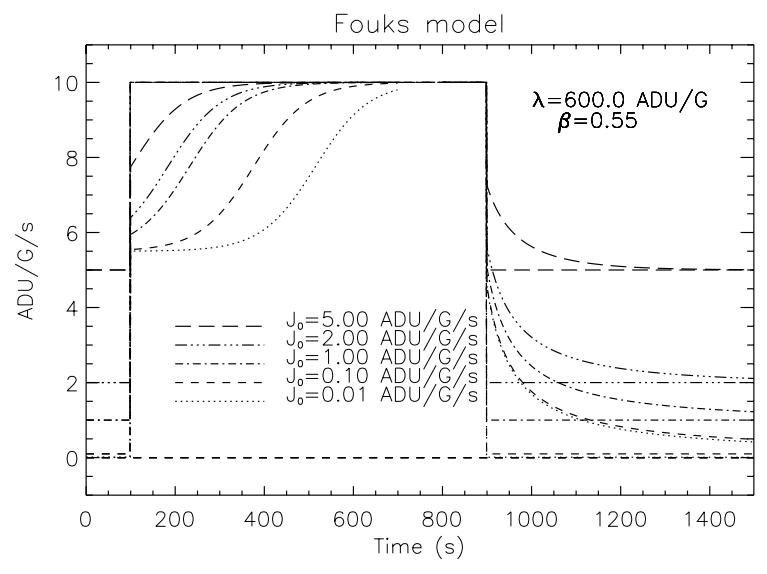

Fig. 4. FS model response to upward and downward steps of flux, from Eq. (1) (see also Fig. 8 in Schubert \& Fouks 1995). Upward steps of flux from a constant level $J_{0}$ to a constant level $J_{1}$ occur at time $t=100 \mathrm{~s}$ and downward steps from $J_{1}$ to $J_{0}$ at $t=900 \mathrm{~s}$. We have taken: $J_{0}=0.01,0.1,1.0,2.0$ and 5.0 ADU/G/s, and $J_{1}=10 \mathrm{ADU} / \mathrm{G} / \mathrm{s}$. For all these simulations, the values of the parameters $\beta$ and $\lambda$ are constant. We see that Eq. (1) is very sensitive to the initial level $\left(J_{0}\right)$ for the upward steps: curves from 0.01, 0.1 and 1.0 ADU/G/s are very different. When the dark level is poorly estimated, such non linear effect can allow us to correct the value of $J_{0}$

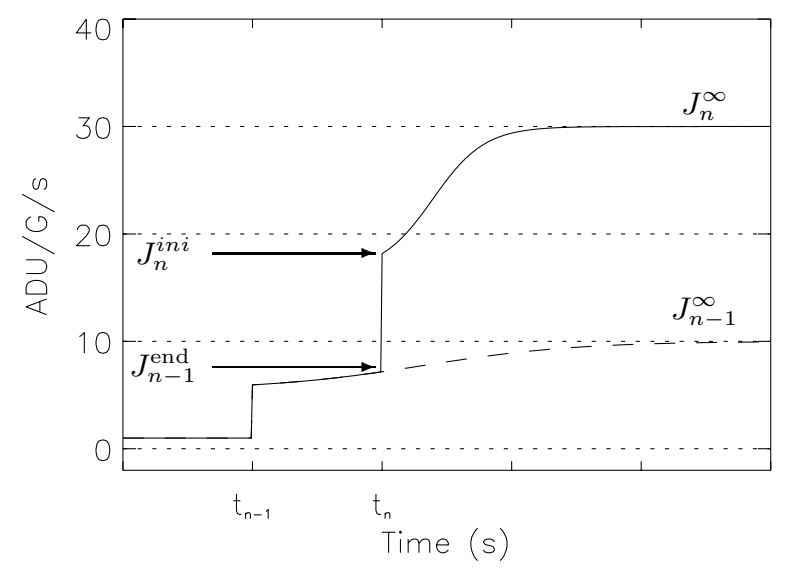

Fig. 5. Response to two successive upward steps of flux (at time $t_{n-1}=100 \mathrm{~s}$ and $t_{n}=200 \mathrm{~s}$ respectively), corresponding to three blocks of constant input fluxes (number $n-2, n-1$ and $n$ respectively). We have taken $\beta=0.55, \lambda=600$. ADU $/ \mathrm{G}$, $J_{n-2}^{\text {end }}=J_{n-2}^{\infty}=1$. ADU $/ \mathrm{G} / \mathrm{s}, J_{n-1}^{\infty}=10$. ADU $/ \mathrm{G} / \mathrm{s}$ and $J_{n}^{\infty}=$ 30. ADU/G/s. This figure is adapted from Fouks \& Schubert (1995)

Finally, we obtain from Eq. (4) and Eq. (5):

$J_{n}(t)=\beta J_{n}^{\infty}+\ldots$

$\frac{(1-\beta)\left(J_{n-1}^{\text {end }}-\beta J_{n-1}^{\infty}\right) J_{n}^{\infty}}{\left(J_{n-1}^{\text {end }}-\beta J_{n-1}^{\infty}\right)+\left((1-\beta) J_{n}^{\infty}-\left(J_{n-1}^{\text {end }}-\beta J_{n-1}^{\infty}\right)\right) \mathrm{e}^{-\left(t-t_{n}\right) J_{n}^{\infty}} / \lambda}$.

$J_{n}(t)$ depends on $J_{n}^{\infty}, J_{n-1}^{\infty}$ and $J_{n-1}^{\text {end }}$ (Fig. 5).

If we take: $J_{n-1}^{\infty}=J_{n-1}^{\text {end }}=0$, we have $\forall t>0, J_{n}(t)=$ $\beta J_{n}^{\infty}$, and $\forall n^{\prime}>n, J_{n^{\prime}}(t)=\beta J_{n^{\prime}}^{\infty}$. Negative values for 


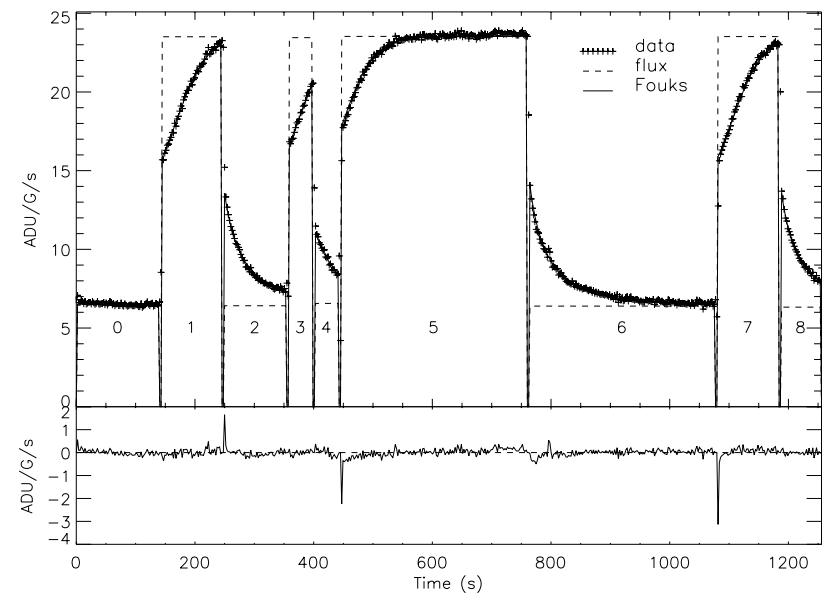

Fig. 6. Block by block correction. These data are taken during the Performance Verification phase (Revolution number 16). The zodiacal background was continuously observed, and the input sky brightness modulated between two values by moving the filter wheel. Upper panel: Mean value of the $11 \times 11$ central square as a function of the time. The integration time per frame was $2.1 \mathrm{~s}$. The unknown flux $J_{n}^{\infty}$ is fitted for each block with constant $(\beta, \lambda)$ parameters. The adjusted input fluxes are over plotted (dashed line), together with the response computed with the FS model (solid line). Lower panel: residue. Evidence of a not perfect fitting can be seen at the beginning and at the end of block 5

$J_{n-1}^{\infty}$ are not physical and would give, after the instantaneous jump, a monotonous decrease with a vertical asymptote located after the jump (its position can be calculated from Eq. (1)).

\subsection{Adjustment of the model}

We have adjusted the model of the response on experimental data, both on the averaged response within the $11 \times 11$ central square and for individual pixels. We have used data with limited flux steps, since the response for steps greater than $\sim 150 \mathrm{ADU} / \mathrm{G} / \mathrm{s}$ is too fast to give accurate values (typically a few integrations).

We illustrate the results with data already presented in Sect. 2. The signal was stabilized before the flux changes. The values $J_{0}^{\infty}$ and $J_{1}^{\infty}$ are known. We have three free parameters to fit Eq. (1) with the data, the two parameters $\beta$ and $\lambda$, and an offset level. To add an offset is mandatory for some observations (especially for the ground base data) since the absolute dark level was poorly estimated while the response is very sensitive to the initial level before the flux step, therefore to the absolute value of the dark level (see Sect. 3.2 and Fig. 4). The three parameters are adjusted by least square fitting $\left(\chi^{2}\right.$ criterion with constant error). We see on the examples we present (Figs. 2 and 3) that the model remarkably reproduces both upward and downward steps. Other steps have been adjusted, with higher and lower amplitudes, with a similar accuracy. Especially, the position of the inflection point of upward steps is accurately reproduced.

We have also made adjustments when the signal was not stabilized before the changes of flux using Eq. (6) to estimate the value of $J^{\infty}$ for each block (one block corresponding to a constant configuration of the whole system, see Sect. 4). We assume that $\beta$ and $\lambda$ are constant over the whole observation. We see in Fig. 6 that the precision is $\sim 1 \%$. We have the same kind of results for different datasets taken during the whole life of the satellite. We have also checked that the theoretical relationship $\tau=\lambda / J^{\infty}$ predicted by Suris \& Fouks (1980) is verified by the data, for uniform illumination of the detector and for upward and downward steps of flux in the range of a few $\mathrm{ADU} / \mathrm{G} / \mathrm{s}$ to $\sim 500 \mathrm{ADU} / \mathrm{G} / \mathrm{s}$.

\subsection{Pixel to pixel variations of the parameters $(\beta, \lambda)$}

The fitting method described in the previous section has been applied for the $32 \times 32$ pixels of the detector in order to derive for each pixel the values of $\beta$ and $\lambda$ for uniform illumination. Practically, we have used long-term observations of the zodiacal background taken successively with two filters. First the data have been dark corrected, then glitches have been removed using a temporal median filtering which detect all pixels deviating from the running median value by more than a threshold value. In order to remove glitches still contained in the data (especially those with significant memory effects, Désert 1999), two pass fitting is used. For the second pass, pixels deviating from the running averaged value of more than $3 \sigma$ are discarded. The correction of glitches with negative tail is much more difficult and manual removing is necessary for a limited number of case. In any case, this two pass fitting does not discard all glitches with memory effects.

The FS model is not symmetrical. The adjustment of $\beta$ and $\lambda$ is more accurate for upward steps than downward steps, because the $\chi^{2}$ values present in the $(\beta, \lambda)$ plane a steeper minimal value for upward steps than downward steps. On simulated noisy data, we have obtained a precision of $\Delta \beta / \beta \sim 0.02$ and $\Delta \lambda / \lambda \sim 0.03$ for upward steps, and only $\Delta \beta / \beta \sim 0.2$ and $\Delta \lambda / \lambda \sim 0.1$ for downward steps.

We have used upward steps of flux on quasi uniform illumination (essentially zodiacal emission) with $J_{0}^{\infty}$ from 5 to $25 \mathrm{ADU} / \mathrm{G} / \mathrm{s}$ and a ratio $J_{1}^{\infty} / J_{0}^{\infty}$ in the range $2-5$. This range is unfortunately limited for in-flight data by the zodiacal emission characteristics. Values of $J_{0}^{\infty}$ lower than $10 \mathrm{ADU} / \mathrm{G} / \mathrm{s}$ give poor adjustments, likely due to uncertainties in the dark level.

Actually, six independent data sets (from scientific observations of diffuse clouds taken during revolutions number $129,321,590,658,684$ and 772 ) have been extensively 

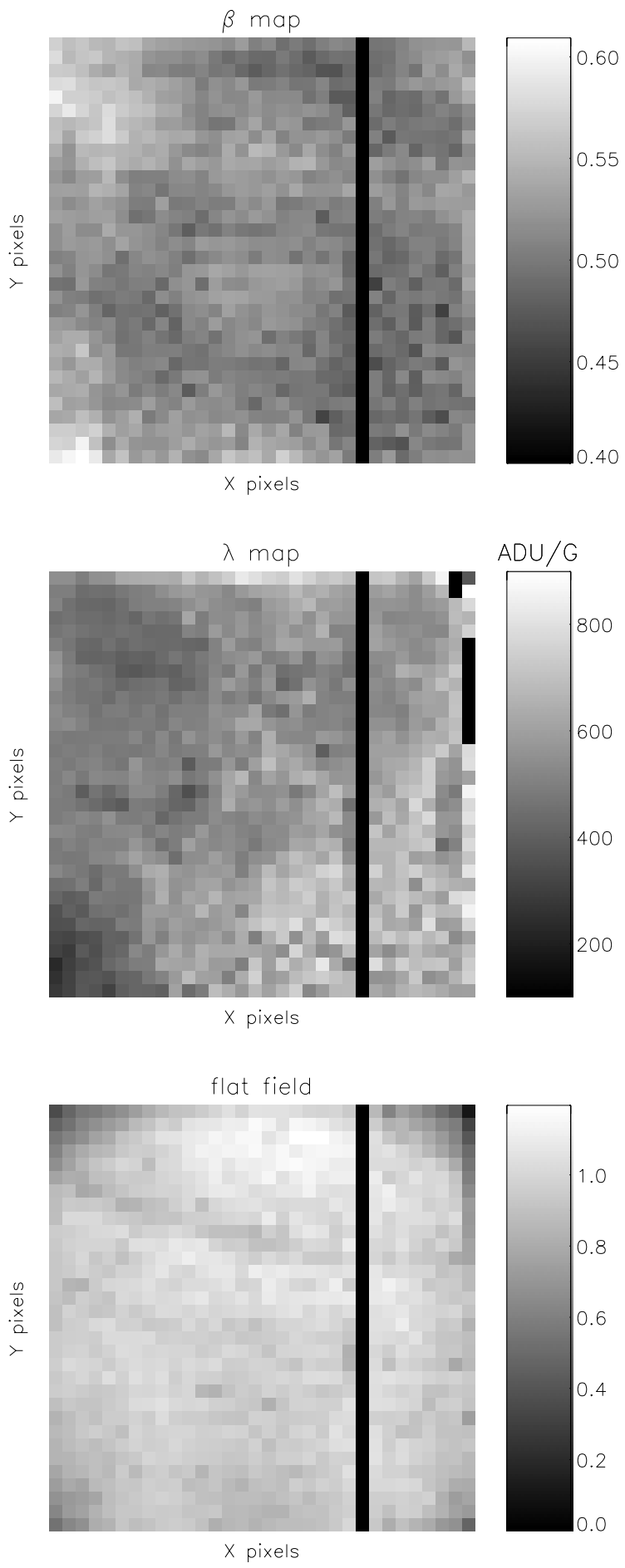

Fig. 7. Final $(\beta, \lambda)$ maps fitted on upward steps from six independent data sets. Values with poor $\chi^{2}$ are discarded before computing the mean value. One column was disconnected during the whole mission. The lower map presents the spatial variations of the response (flat field) to a uniform illumination

analyzed. Because of (1) glitches with memory effects, (2) oscillations in the response curve and (3) in some cases
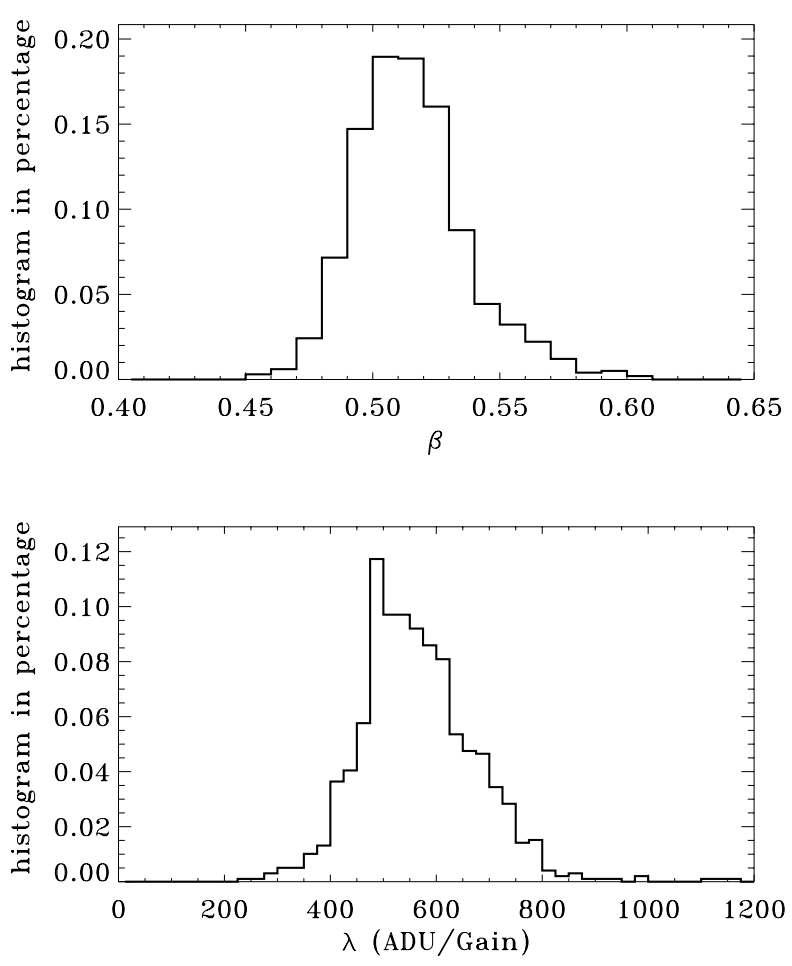

Fig. 8. Histogram of $\beta$ (upper panel) and $\lambda$ (lower panel) for the $32 \times 32$ pixels and from data of Fig. 7

low signals at the edge of the detector due to the optical vignetting, the values of $\beta$ and $\lambda$ cannot be accurately estimated for typically $\sim 15 \%$ of the pixels in an observation. About 5 to $10 \%$ of the pixels in each data set present oscillations during the stabilization after the upward steps. Most of these pixels are close to the edges or the corners of the detector (Abergel et al. 1999). The FS model cannot describe such behavior, and only $\beta$ can be accurately estimated. These oscillations will be discussed in Sect. 5.6.

We have computed six independent maps of $\beta$ and $\lambda$ from the six data sets we use. On these maps, we have pixel to pixel variations which are significant. They are reproducible on the six independent data sets. The final maps of $\beta$ and $\lambda$ are obtained by averaging the six maps taking into account for each pixel the $\chi^{2}$ value for each data set and discarding values by $\sigma$ clipping. The final values of $\beta$ have an absolute accuracy better than \pm 0.02 for $95 \%$ of the pixels. For the final values of $\lambda$, the accuracy is better than $\pm 20 \mathrm{ADU} / \mathrm{G}$ for $\sim 50 \%$ of the pixels (the lower left part of the map presented in Fig. 7), while for $\sim 10 \%$ of the pixels the accuracy is above $50 \mathrm{ADU} / \mathrm{G}$. The final maps are presented in Fig. 7, compared to the map of the spatial variation of the response for uniform illumination (the flat field). Histograms are plotted in Fig. 8.

The accuracy on the final maps of $\beta$ and $\lambda$ is limited because (1) only six data sets have been used with a limited range of the $J_{1}^{\infty} / J_{0}^{\infty}$ ratio, (2) at low $J_{1}^{\infty} / J_{0}^{\infty}$ ratio 
the FS model is not very sensitive to $\lambda$, (3) several pixels are affected by glitches, (4) for some pixels only one or two independent values have been used and (5) uncertainties in the dark level.

The mean values of $\beta$ and $\lambda$ are equal to $\bar{\beta}=0.51$ and $\bar{\lambda}=560 \mathrm{ADU} / \mathrm{G}$. The data taken during the groundbased tests have also been extensively used to explore the transient behavior over a wide dynamic range. Different values $(\bar{\beta} \sim 0.6$ and $\bar{\lambda} \sim 400 \mathrm{ADU} / \mathrm{G})$ are obtained due to a different setup of the detector.

\subsection{Consequences for one of the model hypotheses}

One important assumption of the FS model is (Vinokurov \& Fouks 1991):

$\ln \left(\frac{J_{1}^{\infty}}{J_{0}^{\infty}}\right)<<\frac{E_{0}}{E_{j}}$

where $E_{0}$ is the electrical field between the contacts and $E_{j}$ is a parameter which characterizes the quality of the contacts. We have no information about $E_{j}$ but we can derive it from the parameters $\beta$ and $\lambda$, since:

$E_{j}=\frac{\beta \lambda}{2 \epsilon \epsilon_{0}}$.

We have found $E_{j} \sim 5.10^{3} \mathrm{~V} / \mathrm{m}$ and $E_{0} \sim 4.10^{4} \mathrm{~V} / \mathrm{m}$ then the condition 7 is satisfied. For PHOT-S, Schubert (1995) indicates $E_{j} \sim 2.10^{3} \mathrm{~V} / \mathrm{m}$ and $E_{0} \sim 1.10^{5} \mathrm{~V} / \mathrm{m}$.

\section{Transient correction: Inversion method}

\subsection{Introduction}

We have seen in the previous section that the FS model allows an adjustment of the response curves with an accuracy better than $1 \%$ for more than $90 \%$ of the pixels for uniform illuminations on the whole useful dynamic range. The two parameters of the model $(\beta$ and $\lambda)$ have been adjusted pixel by pixel. No significant temporal variations have been found during the ISO mission. We now present correction methods based on this model.

Basically two methods are possible to correct the data from the transient effects: readout by readout and block by block. One readout corresponds to the output value measured at the end of one integration. One block (typically a few to several hundreds of readouts) corresponds to a constant configuration of the whole system (parameters of the camera, pointing of the satellite). To take into account this information it may help to improve the stability of the correction and the signal to noise ratio.

At the present time and in this paper, we only indicate methods which allow us to correct the data readout by readout. Obviously, we need to assume that during each integration, the input flux is constant. It is wrong during the slew of the satellite during two successive sky positions, or when a change of one parameter of the instrument (lens and filter wheels position) is commanded. Generally, only one or two successive readouts are affected.

\subsection{Readout by readout correction method}

The correction method is based on the FS model described in Sect. 3. We use the same notations as in Eq. (6). We consider a set of $N$ successive readouts $J_{n}^{\text {out }}$, in units of $\mathrm{ADU} / \mathrm{G} / \mathrm{s}$. Each term $J_{n}^{\text {out }}$ can be identified with $J_{n}(t)$ in Eq. (6), if we take $t$ equal to the time of the end of the integration number $n$ which starts at time $t_{n}$ (see Fig. 5). The transient correction consists of computing, for $n$ going from 0 to $N-1, J_{n}^{\infty}$ from the measured responses $J_{n}(t)$. Practically we need to invert Eq. (6) in order to compute $J_{n}^{\infty}$ from $J_{n}(t), J_{n-1}^{\infty}$ and $J_{n-1}^{\text {end }}$. Equation (6) is non-linear because of the exponential term and there is no analytical solution.

We have tested three different methods to find the solution:

- A second order development of the exponential around 0 gives a third order polynomial in $J_{n}^{\infty}$. The closest root to the measured value $J_{n}(t)$ gives the solution. This is the quickest method, but only applicable for low values of $\left(t-t_{n}\right) J_{n}^{\infty} / \lambda$, so of $J_{n}^{\infty}$;

- The monotonic property of Eq. (4) allows us to use an iterating algorithm. First we start by replacing in the exponential $J_{n}^{\infty}$ by $J_{n}(t)$. We obtain a second order equation, so a first estimate of $J_{n}^{\infty}: J_{n, 1}^{\infty}$. Then we replace in the exponential $J_{n}^{\infty}$ by $J_{n, 1}^{\infty}$, and we iterate. This method converges after a few iterations for any steps of flux with no or very limited noise, but practically fails for any steps of flux on experimental data because of the noise level;

- The Müller method (in Numerical Recipes, Press et al. 1992) finds roots of non-linear equations when three initial points are provided. This numerical method is more time consuming.

In order to study the inversion quality and its noise robustness, we have tested these three correction methods on simulated data for different fluxes, different steps of fluxes and different signal to noise ratio. Practically we choose an input flux history: $J_{n \text {,input }}^{\infty}$, for $n$ going from 0 to $N-1$. The detector response $\left(J_{n}(t)\right.$, for $n$ going from 0 to $N-1$ ) is computed, readouts by readouts, using Eq. (6). Then we add a Gaussian noise with a standard deviation up to $\sigma=1 \mathrm{ADU} / \mathrm{G} / \mathrm{s}^{1}$. All the values $J_{n}(t)$ were positive before the noise is added but not necessarily after. For $n$ going from 0 to $N-1$, we compute readout by readout the corrected response $J_{n}^{\infty}$ using the three methods described above. Finally we compare $J_{n \text {,input }}^{\infty}$ and $J_{n}^{\infty}$. Practically we have used the same kind of signal as in Fig. 4, with typically 600 readouts, upward steps at readout 200, downward step at readout 400, an integration time $t_{\text {int }}$ of $2.1 \mathrm{~s}$ for each readout, $\beta=0.55$ and $\lambda=600 \mathrm{ADU} / \mathrm{G}$. The low levels are equal to $0.1,0.5,1,2,5$ and

1 The real noise may depart from a simple Gaussian law especially due to glitches with memory effects. At low levels, the experimental noise is generally lower than $1 \mathrm{ADU} / \mathrm{G} / \mathrm{s}$. 
$10 \mathrm{ADU} / \mathrm{G} / \mathrm{s}$, while high levels are 5, 25, 50, 100, 250, 500 or $1000 \mathrm{ADU} / \mathrm{G} / \mathrm{s}$.

The Müller method gives always the more accurate result even for high steps (typically for $10^{-3}<J_{n}^{\infty} / J_{n-1}^{\infty}<$ $\left.10^{3}\right)$ and strong noise $(\sigma=1 \mathrm{ADU} / \mathrm{G} / \mathrm{s})$. But this method is also the slowest one. Sometimes divergences occur for levels below $1 \mathrm{ADU} / \mathrm{G} / \mathrm{s}$.

The iterating approach is very sensitive to the amplitude of the noise. On the simulations, divergence could occur for any step ratio when $\sigma$ is greater than $\sim 0.1$ $\mathrm{ADU} / \mathrm{G} / \mathrm{s}$. On real data, divergences occur quite often. Thus we conclude that this method has to be discarded.

The method based on a second order development of the exponential around 0 must be used only for limited fluxes $J_{n}^{\infty}$, since the factor $\left(t-t_{n}\right) J_{n}^{\infty} / \lambda$ must be significantly smaller than 1 . This method presents the advantage that at low flux it never diverges, even with noise of high amplitude $(\sigma \sim 1 \mathrm{ADU} / \mathrm{G} / \mathrm{s})$, and is extremely fast.

Practically, we use this last method at small flux, and the Müller one at high fluxes. In any case, the computing time is typically ten times lower than with the old IAS method (Abergel et al. 1999) for an accuracy typically ten times better. On simulations, the accuracy is better than $1 \%$ for each readout; on real data, the accuracy is better than $\sim 3 \%$ for each readout when we have not problem due to glitches, spatial gradients or inaccurate dark levels...

Figures 9 and 10 present two results to illustrate the quality of the correction of real data for uniform illumination. For observations with the Circular Variable Filter $(\mathrm{CVF})$, after correction we see that the data can be used on the whole spectral range. It was generally not the case with previous methods.

\section{Limitations and work in progress}

\subsection{Flux history before the observation}

One critical problem with the correction methods comes from the difficulty to estimate properly the flux history before the first readout of the observation to be processed. Especially at low levels, strong memory effects due to the unknown history may affect the whole observation.

Practically, an observation is made, for all pixels, of $N$ readouts: $J_{n}(t)$, for $n$ going from 0 to $N-1$. Whatever the correction method is, the first corrected value $J_{0}^{\infty}$ is computed from $J_{0}(t), J_{-1}^{\infty}$ and $J_{-1}^{\text {end }}$ (Eq. 6), where $J_{-1}^{\infty}$ and $J_{-1}^{\text {end }}$ are the input flux during the integration before the first one and the measured signal at the end of this integration respectively. We see that the state of the pixel before the first integration (number 0 ) is fully contained in $J_{-1}^{\infty}$ and $J_{-1}^{\text {end }}$ (if stabilization, $J_{-1}^{\infty}=J_{-1}^{\text {end }}$ ). Therefore to correct the response measured by one pixel, it is mandatory to estimate as properly as possible these two numbers.

Actually, two alternative methods can be used to estimate the flux history before the observation:
- If the first readouts seem stabilized, it is assumed that $J_{-1}^{\infty}$ and $J_{-1}^{\text {end }}$ are equal to an averaged value of the first readouts for each pixel;

- If the first readouts are not stabilized then if we can supposed that (1) the flux is constant at the beginning of the observation and during a significant number of integrations and (2) the flux before staring the observation was also constant, then it is possible to apply the fitting techniques presented in Sect. 3.4 to estimate $J_{-1}^{\infty}$ and $J_{-1}^{\text {end }}$.

It is obviously much more difficult to recover a realistic history at low level than at high level because of longer time constants and smaller signal to noise ratio. This point can be critical for CVF observations.

\subsection{Time discontinuities in data set}

In the ideal case, the data should be corrected without any time discontinuity. The observing time of ISO was shared between the four focal plane instruments. However, even when ISOCAM was not the prime observing instrument, it was still active and data were transmitted with a low telemetry rate (Siebenmorgen et al. 1996), except during 6 hours per revolution when the instruments are switched off.

Temporal discontinuities in the data flow come from: (1) turn on at the begin of each revolution, (2) from time to time, telemetry was canceled, (3) problems due to on board software when filter wheel motors are commanded, (4) instantaneous glitches (one or few readouts lost), (5) data splitting due to data proprietary protection.

The transient correction works without any problems induced by glitches (which are actually substituted by a local median value). Points (2) and (3) are equivalent to the glitches problem (4). Point (5) should disappear in the next future since the data can now be processed revolution by revolution.

\subsection{Accuracy of the dark correction}

We have shown in Sect. 3.2 and in Fig. 4 that the FS model is very sensitive to the absolute level. This is critical near the dark level, since the time constants of the response are inversely proportional to the current observed flux. Moreover, 0 is a singular point in the model (we have seen in Sects. 3.2 and 3.3 that if we have for one readout $J^{\infty}=J^{\text {end }}=0$, then the response for all the following readouts is $\left.\beta J^{\infty}\right)$. Negative values may induce long term divergence in the correction. Therefore for observations with low input fluxes (especially with the CVF), the accuracy of the dark correction is critical.

At the present time, a dark image computed using the estimator of the time behavior of the dark signal (Biviano et al. 1998) is subtracted for each readout. In some cases, 

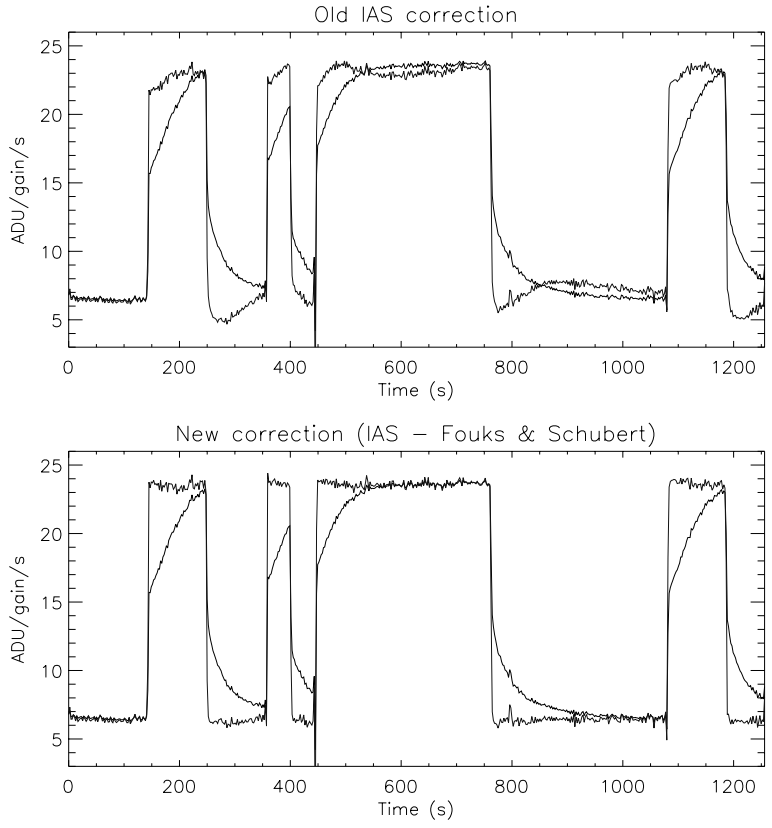

Fig. 9. Corrections of transients with the old IAS method (upper panel) and our new method (lower panel). The data are the same as in Fig. 6

the dark subtraction is not perfect since some negative values may appear. For data containing significant upward steps of flux, the FS model can be used to refine the determination of the absolute dark level.

\subsection{Long term drift}

The correction method we have presented does not correct the long term drift (see Fig. 1 and Sect. 2) which generally affects the data after a positive upward step. The physical origin of this drift is still not understood.

For large scale mapping obtained by successive pointings of the satellite (raster mode), a powerful correction method is proposed by Miville-Deschênes (1999). This method uses the redundancy of the observation since in raster mode a significant fraction of the sky positions is observed by different pixels at different times.

\subsection{The problem of point sources}

When the observed field contains high spatial gradients, the FS model cannot describe the observations and the corrections we propose in this paper generally fail. This is critical for point sources since the responses measured for bright sources are actually never properly corrected.

For the same initial and final levels (after stabilization), the response of all pixels of the point source departs from the uniform illumination response. For the brightest pixel the response is (1) generally non monotonous,
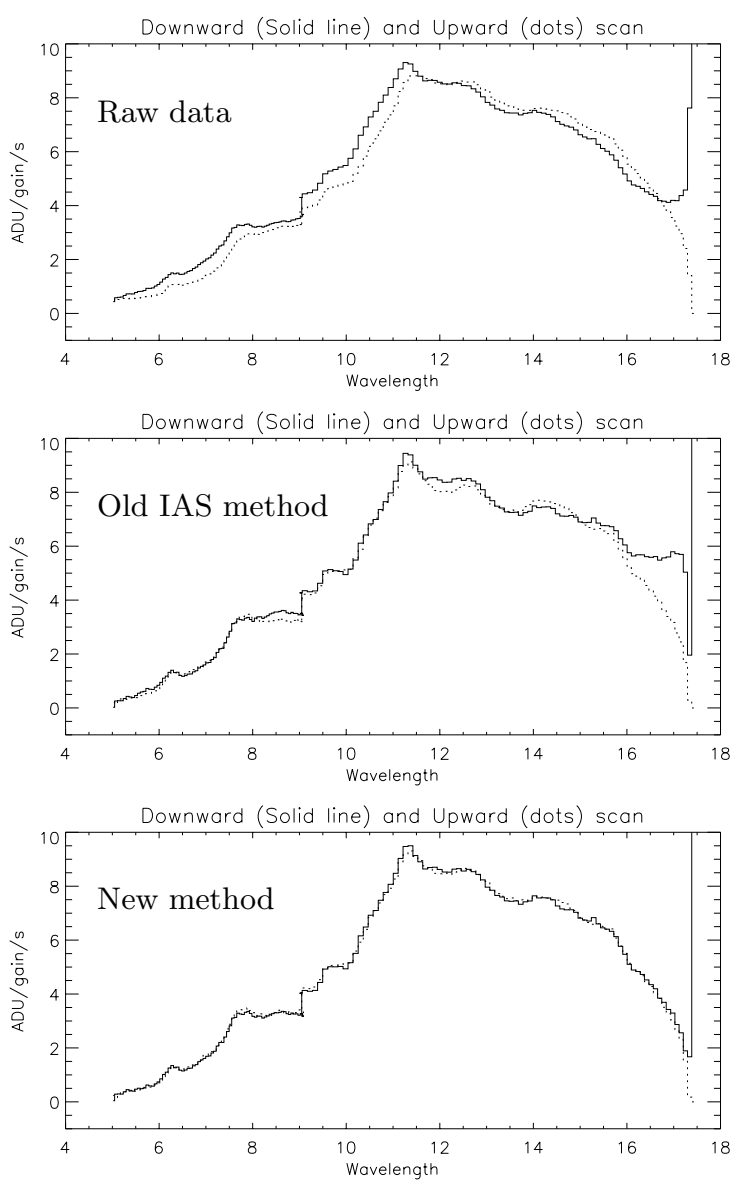

Fig. 10. Comparison between CVF reconstructions with the old IAS method and the new one, for downward and upward scanning. Upper panel: one full scan CVF (downward (solid line) and upward (dots)), original data; middle panel: inversion with the old IAS method; bottom panel: inversion with the new method we propose, based on the Fouks-Schubert model. (1) the shape of the downward scan is correct over the full range, even at the beginning of the observation (we start at $17.5 \mu \mathrm{m}$ ) and (2) upward and downward are quasi superposed over the full range

(2) is faster than the FS one and (3) a peak appears for strong sources (brightest pixel at least $80 \mathrm{ADU} / \mathrm{G} / \mathrm{s}$ above the background). The brighter the source, the higher that peak.

The model we present in this paper is developed for low contrasted illumination of the detector. The cross talk between pixels (Fouks 1992; Fouks et al. 1994) and non linear effects due to a non uniform illumination of the pixel (the PSF is under-sampled for a large fraction of observations) have been neglected. We are currently working on these problems. 


\subsection{Oscillations in the response curve}

We have seen (Sect. 3.5) that for typically $10 \%$ of the pixels, the FS model does not allow us to describe properly the response because of temporal oscillations in the response curve. Most of these pixels ${ }^{2}$ are close to the detector's edges and corners (Abergel et al. 1999). In a first order, the higher the amplitude of the flux step, the higher the amplitude of the oscillations. For these pixels, the correction obviously fails. However, at low levels (few tens of $\mathrm{ADU} / \mathrm{G} / \mathrm{s}$ ) the mean error remains generally limited (a few $\%$ ) after transient correction.

One possible explanation could be cross talk between pixels since the gradient of charge distribution is higher near the edges than in the central part of the detector. However, Fouks (1997) has suggested an improvement of the FS model to deal with these oscillations without cross talk. But first attempts with this model fail to reproduce the oscillations observed in ISOCAM LW data.

\section{Application of Suris-Fouks-Vinokurov models for other detectors?}

We have shown that the FS model is able to describe very accurately the short term transient for most of the pixels of the LW detector of ISOCAM, for uniform illumination. This remarkable agreement indicates that: (1) the SurisFouks-Vinokurov team has properly described the physics of such low background IR detectors and (2) influences of spurious phenomena (as electrodes effects) in the detector are very limited.

All ISO detectors (LWS, SWS, PHOT and SW-CAM) are also based on extrinsic semiconductors technology working under low illumination. Ground-based tests and in-flight observations show that the non-linear behavior and the temperature dependence of the response curves of these detectors seem perfectly reproducible, as for ISOCAM (same illumination history give the same measurements). Different authors (Suris \& Fouks 1980; Fouks 1981a; Fouks 1981b; Vinokurov \& Fouks 1991; Fouks 1992) have solved the solid state equations for different kinds of extrinsic detectors. One of the strongest hypothesis is the bulk thickness, since with a thin thickness, a hook behavior is predicted.

Variations of the temperature of the detectors could also affect the response curves. For LW-ISOCAM, these variations are always lower than $1 \%$ around the mean value (close to $3 \mathrm{~K}$ ). For ISOPHOT-S1, variations could be of $\simeq 10 \%$ (Schubert 1995) and must be taken into account (Fouks \& Schubert 1995).

The transient behavior of other detectors on ISO should also be described by this kind of model. Therefore a transient correction should also be possible without any

\footnotetext{
${ }^{2}$ For instance, the pixel $(29,11)$ presents systematic overshoot before stabilization, even for limited flux steps.
}

fitting or parameters adjustments. It should allow a significant increase of the final accuracy of processed data.

The current main limitation of these models is they are one dimensional: the pixel surface must be uniformly illuminated and no gradient must exist between neighborhood pixels.

\section{Conclusion}

The main results of the paper are the following:

1. the Fouks-Schubert model allows us to describe the short term transient for LW-ISOCAM data for uniform illumination of the detector. The accuracy is better than $1 \%$ for more than $90 \%$ of the pixels;

2. From independent datasets, we have evidence of spatial variations in the maps of the two parameters of the model $(\beta$ and $\lambda)$. No significant temporal variations of these parameters have been found during the ISO mission;

3. The FS model cannot be applied directly to describe the behavior of point source response and the long term drift;

4. Original and efficient inversion method has been proposed;

5. The theoretical model we use is only one special case of a large family of non linear models. Applications to other ISO detectors must be tested.

We can now correct data taken with the LW channel of ISOCAM with an accuracy of $1 \%$ for low contrasted fields.

Acknowledgements. We thank the different ISO teams at IAS, CEA-Saclay, ESA and IPAC for their outstanding work and continuous support during all phases of the ISO project. A. Coulais also would like to thank the CNES (France) for its financial support.

\section{References}

Abergel A., et al., 1996, A\&A 315, L329

Abergel A., Miville-Deschêne M.A., Désert F.X., et al., 1999 (accepted in Exp. Astron.)

Abergel A., Pérault M., 1994, Flight model LW channel: noise due to transients, Technical report, IAS Lab.

Agnèse P., Lucas C., Maillart P., et al., 1989, Proc. SPIE 1070, 124

Biviano A., et al., 1998, The ISOCAM Dark Current Calibration Report, Technical report, ISOCAM

Blommaert J., 1998, ISOCAM photometry report, Technical report, ESA Vilspa, Spain

Cesarsky C.J., 1992, in: Encrenaz T. and Kessler M. (eds.), Infrared Astronomy with ISO. L'astronomie infrarouge et la mission ISO., Les Houches, France, June 13-27, 1991, Nova Science, Commack, NY, pp. 31-50

Cesarsky C.J., et al., 1996, A\&A 315, L32 
Church S.E., Griffin M.J., Ade P.A.R., et al., 1992a, in: ESA SP-356 Photon Detectors for Space Instrumentation, pp. $255-260$

Church S.E., Price M.C., Griffin M.J., et al., 1992b, in: ESA SP-356 Photon Detectors for Space Instrumentation, pp. 261-264

Delattre C.J., 1996, technical report

Désert F.-X., 1999, A modified IAS model for ISOCAM LW transient correction, Technical report, LAOG, Version 2.0

Fouks B.I., 1981a, Sov. Phys. Semicond. 15(9), 974

Fouks B.I., 1981b, Sov. Phys. Semicond. 15(9), 980

Fouks B.I., 1992, in: ESA SP-356 Photon Detectors for Space Instrumentation, pp. 167-174

Fouks B.I., 1996, Proc. SPIE 2790, 38

Fouks B.I., 1997, Proc. SPIE 3122, 441

Fouks B.I., Kocherov V.F., Taubkin I.I., et al., 1994, Proc. SPIE 2269, 727

Fouks B.I., Schubert J., 1995, Proc. SPIE 2475, 487

Ganga K., Helou G., Hurt R., et al., 1998a, ISO/CAM transient removal at IPAC, Technical report, IPAC Lab.

Ganga K., Kong M., Jing L., Van Buren D., 1998b, Transient removal with the IPAC method, Technical report, IPAC Lab.

Groezinger U., Kirches S., Lemke D., et al., 1992, in: ESA SP-356 Photon Detectors for Space Instrumentation, pp. 329-333

Kessler M.F., et al., 1996, A\&A 315, L27
Lari C., 1997, IRA model for ISOCAM LW transient correction, Technical report, IRA

Miville-Deschênes M.-A., Boulanger F., Abergel A., Bernard J.-P., 1999, Original data processing for ISOCAM raster imaging of extended emission (accepted in A\&A)

Pérault M., Desert F.X., Abergel A., et al., 1994, Opt. Eng. 33, 762

Press W., Teukolsky S., Vetterling W., Flannery B., 1992,

Numerical Recipes in FORTRAN, second edition. Cambridge University Press

Schubert J., 1995, Ph.D. Thesis. Max Planck-Institut für Astronomie, Heidelberg

Schubert J., Fouks B.I., Lemke D., Wolf J., 1995, Proc. SPIE 2553, 461

Schubert J., Roth G., Wolf J., et al., 1994, Proc. SPIE 2268, 283

Siebenmorgen R., et al., 1996, A\&A 315, L169

Suris R., Fouks B.I., 1978, Sov. Phys. Semicond. 12(12), 1380

Suris R., Fouks B.I., 1980, Sov. Phys. Semicond. 14(8), 896

Tiphène D., Rouan D., Epstein G., Le Coupanec P., 1999 (accepted in Exp. Astron.)

Vinokurov L.A., Fouks B.I., 1991, Sov. Phys. Semicond. 25(11), 1207

Wensink J.W., Luinge W., Beintema D., et al., 1992, in: ESA SP-356 Photon Detectors for Space Instrumentation, pp. 339-344 